\title{
Telovelar Approach to Pediatric Fourth Ventricle Tumors: Feasibility and Outcome
}

\author{
Ahmed ATALLAH ${ }^{1}$, Mohamed Reda RADY ${ }^{1}$, Hazem Mostafa KAMAL ${ }^{1}$, Noha EL-MANSY², \\ Mohamed F.M. ALSAWY ${ }^{1}$, Ahmed HEGAZY ${ }^{1}$, Mohamed Shehab Saad ZAGHLOUL ${ }^{1}$ \\ ${ }^{1}$ Cairo University, Faculty of Medicine, Department of Neurosugery, Cairo, Egypt \\ ${ }^{2}$ Cairo University, Faculty of Medicine, Department of Diagnostic and Interventional Radiology, Cairo, Egypt
}

Corresponding author: Ahmed ATALLAH ahmed.atallah09@kasralainy.edu.eg, dr.atallah81@gmail.com

\section{ABSTRACT}

AIM: To describe our institution's experience with the telovelar approach as well as factors governing radicality and outcome for pediatric fourth ventricle tumors.

MATERIAL and METHODS: During March 2015-January 2017, 44 children with fourth ventricular tumors were operated using the telovelar approach. Radicality, neurological outcome, complications, and survival rates were evaluated. Statistical analysis was performed to determine factors associated with radicality and neurological outcome.

RESULTS: Gross tumor removal was achieved in 37 patients (84.1\%). All patients required cerebrospinal fluid (CSF) diversion through a ventriculoperitoneal shunt. Six patients (13.6\%) developed cerebellar mutism, 4 patients (9.1\%) developed bulbar paralysis (lower cranial nerve palsy), and 13 patients $(30.2 \%)$ had tumor recurrence. Medulloblastoma was associated with the highest risk of cerebellar mutism $(p=0.040)$. A poor prognosis (according to overall and progression-free survival rates) was associated with subtotal resection $(p=0.020)$, large cell/anaplastic medulloblastoma and anaplastic ependymoma ( $p=0.038)$, highrisk medulloblastoma $(p=0.005)$, and CSF seeding $(p<0.001)$.

CONCLUSION: The telovelar approach provides an adequate anatomical exposure of the fourth ventricle and allows early visualization and protection of its floor (brainstem). It facilitates adequate radicality and is associated with a lower incidence of cerebellar mutism and other approach-related complications. The working angle, particularly to the rostral part of the fourth ventricle, can be increased by removing the posterior arch of the atlas.

KEYWORDS: Pediatric, Fourth ventricle, Posterior fossa, Telovelar approach, Cerebellar mutism

\section{INTRODUCTION}

$\mathrm{I}$ ts direct relation to the brainstem and deep location anterior and ventral of the cerebellum make access to the fourth ventricle challenging (24). The transvermian approach to the fourth ventricle with splitting of the inferior vermis may result in caudal vermis syndrome, cerebellar mutism, or injury of the dentate nuclei resulting in head nodding, truncal ataxia, and nystagmus $(2,11,21,30)$.
The telovelar approach can be used as the standard approach for almost all fourth ventricle lesions. However, to date, the clinical series reporting on the pros and cons of this approach do not provide sufficient data to adequately evaluate the outcome (26).

\section{MATERIAL and METHODS}

This prospective study included 44 male and female patients with newly diagnosed fourth ventricular tumor treated at \begin{tabular}{ll}
\hline Ahmed ATALLAH & (D) : 0000-0003-4020-0501 \\
Mohamed Reda RADY & (D) : $0000-0003-1971-6933$ \\
Hazem Mostafa KAMAL & (D) : $0000-0001-5441-5779$ \\
Noha EL-MANSY & (D) : $0000-0001-5794-5046$
\end{tabular}
Mohamed F.M. ALSAWY

Ahmed HEGAZY

Mohamed Shehab Saad ZAGHLOUL
(D) : 0000-0002-1481-2494

(D) : 0000-0003-2878-4137

(D) : 0000-0002-0676-9772 
Cairo University Children's Hospital, Egypt, during March 2015-January 2017. Patients with exclusively cerebellar or cerebellopontine angle lesions, recurrent, and residual lesions were excluded. All legal guardians of patients provided written informed consent.

The patients were assessed for their ventricular size (hydrocephalus) through preoperative imaging [computed tomography (CT) and magnetic resonance imaging (MRI)]. All patients had obstructive hydrocephalus, for which ventriculoperitoneal (VP) shunts were inserted.

Routine removal of the posterior arch of the atlas approximately $0.5 \mathrm{~cm}$ from the midline on each side was performed in all cases before the occipital bone flap was elevated. The steps of the telovelar approach were followed with dissection of the arachnoid membrane bilaterally along the tonsillomedullary and uvulotonsillar fissures, allowing for segregation and safer retraction of the cerebellar tonsils (unlocking of tonsils). This gave access to the tela choroidea, which was opened by sharp dissection before the inferior medullary velum was incised. Performing these steps bilaterally allows wide access to the entire fourth ventricle and total tumor resection. In large tumors, central debulking was performed first. The tonsils were retracted slightly upward using retractors. Early identification of the tumor-brainstem interface enabled the protection of the brainstem with a cottonoid. Total tumor excision was the primary goal of surgery, leaving a thin tumor sheet on the ventricle floor only in cases with brainstem invasion (without entering the brainstem). Head flexion together with atlas arch removal facilitated the exposure of the entire length of the ventricular floor up to the Sylvian aqueduct.

Postoperatively, all patients underwent a full neurological examination with an emphasis on posterior fossa syndromes, particularly whether they had complete or partial cerebellar mutism syndrome with determination of the exact components involved (linguistic, neuro-behavioral, and/or motor). Additionally, the examination assessed any delayed cerebrospinal fluid (CSF) collection and CSF leaks.

Non-contrast CT of the brain was performed for all patients within 24 hours of the operation to exclude hematoma and significant pneumocephalus and to assess ventricular size. $\mathrm{MRI}$ of the brain was performed within 48 hours to evaluate the extent of tumor resection. During follow-up, MRls of the brain and spine without and with contrast were performed every 3 months in the first year and every 6 months thereafter to detect any tumor recurrence and/or CSF seeding.

Histopathological examination of the biopsy material and grading according to WHO classification were performed for all patients. Cytological examination was performed on CSF samples obtained no earlier than 2 weeks postoperatively to assess CSF seeding.

All patients, except those with pilocytic astrocytoma grade I (GI), received adjuvant radio- and/or chemotherapy according to their histopathological diagnosis and their risk stratification in case of medulloblastoma.
The outcome was assessed in all patients on the following occasions: immediately postoperative, at discharge, 3 weeks postoperatively, and every 3 months thereafter.

SPSS program (SPSS Inc., Chicago, IL, USA) was used for statistical analysis. Overall survival (OS) was defined as the duration (in months) between tumor removal and final followup or death. Progression-free survival (PFS) was defined as the duration (in months) between tumor removal and first relapse at any site, final follow-up, or death.

The following factors were evaluated with regard to their prognostic value: age ( $<5$ vs. $5-18$ years), gender, metastatic stage (M0 vs. $\mathrm{M}+$ ), extent of resection [gross tumor resection (GTR) vs. subtotal tumor resection (STR)], risk stratification of medulloblastoma [(MB) (standard risk (SR) vs. high risk (HR)], pathological type, tumor site (with vs. without fourth ventricle floor infiltration), and tumor size ( $<5$ vs. $>5 \mathrm{~cm}$ in maximal diameter). Survival analysis (PFS and OS) was performed for the various outcome factors (univariate analysis) using Kaplan-Meier statistics. Cox multivariate regression was used to determine the effect of important variables on OS and PFS. $\mathrm{p}<0.05$ was considered statistically significant.

\section{RESULTS}

\section{Patients Characteristics}

There were 27 male and 17 female patients (1.6:1) (Table I); the chief presenting symptoms and signs are listed in Table II.

Patients aged 5-18 years showed no significant difference in PFS from those aged $<5$ years (2-year PFS $70.6 \%$ vs. $62.7 \%$, $\mathrm{p}=0.508$ ) (Table III).

\section{Tumor Characteristics}

Tumors were classified according to their size (maximal diameter in $\mathrm{cm}$ ) into two groups: $<5 \mathrm{~cm}$ and $>5 \mathrm{~cm}$ as shown in Table I.

\section{Tumor Location and Extent of Resection}

Tumor location was defined according to preoperative imaging and surgical reports. The tumors were divided into two subtypes (Table I). The first subtype comprised tumors with fourth ventricle (V4) floor infiltration (attachment to brain stem); the second subtype comprised tumors without V4 floor infiltration. The two groups showed no statistically significant difference in outcome ( $p=0.947$ for PFS and $p=0.690$ for OS). GTR was defined as either a tumor residue $<1.5 \mathrm{~cm}^{2}$ in the postoperative MRI, no visible tumor remaining or a thin layer of tumor left on the brain stem. Of the 24 patients whose tumors were found to invade or be attached to the brainstem, GTR could be achieved in 17 patients (70.8\%). In the remaining patients $(29.2 \%)$, STR was performed because complete resection was prevented by tumor attachment to or invasion of the brainstem $(p=0.008)$ (Table I). Patients who underwent GTR had a better outcome than those who underwent STR (2-year PFS 93.3\% vs. 18.7\%, $p=0.020$ ). 


\section{Histopathological Types}

The pathological subtypes of the tumors in our study are listed in Table I. Patients with pilocytic astrocytoma (AS) Grade II (GII), and ependymoma (EP) Gll had no progression during the follow-up period and the best outcome (2-year PFS 100.0\%). Patients with classical medulloblastoma (CMB) exhibited a better PFS and OS than those with desmoplastic-nodular medulloblastoma (DNMB; 2-year PFS $66.0 \%$ vs. $60.0 \%$ respectively, $\mathrm{p}=0.038)$. Large cell/anaplastic medulloblastoma (LC/A MB) and EP Grade III (GIII) patients had the worst prognosis (2-year PFS 33.3\%).

Table I: Clinical, Demographic and Surgical Data of Study Cases

\begin{tabular}{cc}
\hline Characteristic & No. of patients \\
\hline $\mathrm{n}(\%)$ \\
\hline
\end{tabular}

Age (years):

Mean; 6.05 (range 2 to 15 )

\begin{tabular}{cl}
\hline$<5$ years & $17(38.6)$ \\
\hline $5-18$ years & $27(61.4)$ \\
\hline Gender & $27 \mathrm{M} / 17 \mathrm{~F}$ \\
\hline Tumor Size & \\
\hline$<5 \mathrm{~cm}$ & $15(34.1)$ \\
\hline$>5 \mathrm{~cm}$ & $29(65.9)$ \\
\hline
\end{tabular}

Tumor location

\begin{tabular}{ll}
\hline With V4 floor infiltration & $24(54.5)$ \\
\hline Without V4 floor infiltration & $20(45.5)$ \\
\hline
\end{tabular}

\section{Extent of tumor resection}

\begin{tabular}{cc}
\hline GTR & $37(84.1)$ \\
\hline STR & $7(15.9)$ \\
\hline
\end{tabular}

\section{Pathological subtypes}

\begin{tabular}{lc}
\hline Classic (CMB) & $14(31.8)$ \\
\hline Desmoplastic/nodular (DNMB) & $5(11.4)$ \\
\hline Large cell/anaplastic (LC/A MB) & $8(18.2)$ \\
\hline Ependymoma GII & $4(9.1)$ \\
\hline Anaplastic ependymoma GIII & $3(6.8)$ \\
\hline Pilocytic GI (PA GI) & $9(20.5)$ \\
\hline Grade II, with piloid features & $1(2.3)$ \\
\hline Risk stratification of medulloblastoma & 14 / 27 (51.9) \\
\hline Standard (Average) risk (SR) & $13 / 27(48.1)$ \\
\hline High risk (HR)
\end{tabular}

F: Female, GTR: gross total resection, M: Male, STR: subtotal resection, V4: fourth ventricle.

\section{Postoperative Complications}

The main postoperative complications were (in decreasing order of frequency) neck pain, pseudomeningocele, CSF infection, cerebellar mutism, bulbar palsy, and subdural collection (Table II).

CSF infection occurred in seven patients (15.9\%). They were managed by shunt removal and external ventricular drain insertion until infection subsided, followed by insertion of a new VP shunt. One patient developed severe ventriculitis and septicemia and died.

Bilateral subdural fluid collection occurred in four patients (9.1\%): two were managed conservatively and two required evacuation and upgrading of their medium pressure VP shunts to high pressure shunts.

\section{Cerebellar Mutism Analysis}

Cerebellar mutism (CM) occurred in six patients (13.6\%), and all of them had medulloblastomas (four patients had CMB and two had LC/A MB). The onset of mutism varied from 2 to 3 days postoperatively [mean: $2.50 \pm$ (standard deviation) 0.548 days].

Table II: Clinical Data of Study Cases

\begin{tabular}{lc}
\hline Charactristic & No. of patients (\%) \\
\hline Presenting Symptoms and Signs & \\
\hline Hydrocephalus & $44(100.0)$ \\
\hline Headache \& Crying & $43(97.7)$ \\
\hline Abnormal gait & $31(70.5)$ \\
\hline Vomiting & $30(68.2)$ \\
\hline Papilledema & $29(65.9)$ \\
\hline Blurring of Vision & $28(63.6)$ \\
\hline Diplopia & $14(31.8)$ \\
\hline Cranial nerve palsy & $8(18.2)$ \\
\hline Head tilt & $8(18.2)$ \\
\hline Bulbar symptom & $3(6.8)$ \\
\hline Ataxia & $2(56.8)$ \\
\hline
\end{tabular}

Mainly encountered postoperative complications

\begin{tabular}{lc} 
Neck pain & $19(43.2)$ \\
\hline Psedomeningocele & $12(27.3)$ \\
\hline CSF infection & $7(15.9)$ \\
\hline Cerebellar Mutism & $6(13.6)$ \\
\hline Bulbar palsy & $4(9.1)$ \\
\hline Subdural collection & $4(9.1)$ \\
\hline
\end{tabular}


Four patients $(9.1 \%)$ had mild to moderate mutism with a decrease in verbal output, hypotonia, and oropharyngeal dysfunction, leading to dysphagia and decreased oral intake. In these four cases, gradual spontaneous improvement occurred within 2-6 months postoperatively, with residual dysarthria. The other two patients $(4.5 \%$ of all patients) suffered from severe posterior fossa syndrome with all three components (linguistic, motoric, and neurobehavioral). These two patients had LCA MB and died from disease progression later ( 6 and 8 months after excision, respectively).
Univariate analysis showed a statistically significantly higher risk of $\mathrm{CM}$ for medulloblastomas than for other tumor types $(p=0.040)$, but no significant differences were observed for age $(p=0.380)$, gender $(p=0.662)$, tumor size $(p=0.394)$, and the extent of resection $(p=0.586)$.

\section{Postoperative Bulbar Palsy Analysis}

Three patients (6.8\%) had bulbar symptoms starting preoperatively and four $(9.1 \%)$ had new-onset bulbar symptoms. Of them, five had LC/A MB and two had EP GIII. Two patients had mild to moderate choking that improved

Table III: Two-Year PFS and OS Univariate Analysis in the Different Subgroups

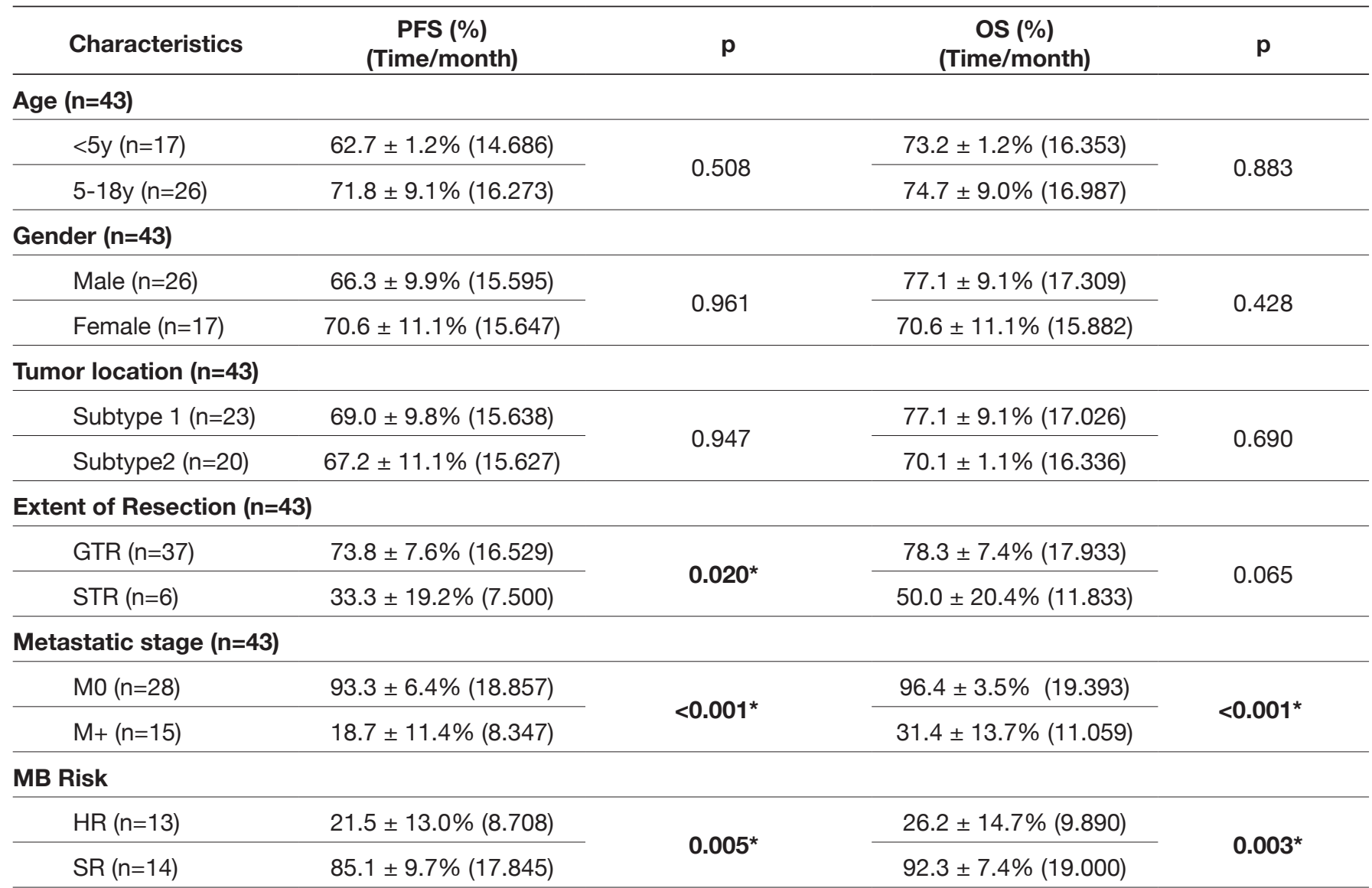

Pathology $(n=43)$

\begin{tabular}{|c|c|c|c|}
\hline CMB $(n=14)$ & $65.7 \pm 14.4 \%$ & \multirow{7}{*}{$0.038^{*}$} & $71.8 \pm 1.4 \%$ \\
\hline DNMB $(n=5)$ & $60.0 \pm 21.9 \%$ & & $80.0 \pm 17.9 \%$ \\
\hline EP GIII ( $n=3)$ & $33.3 \pm 27.2 \%$ & & $66.7 \pm 27.2 \%$ \\
\hline LC/A MB $(n=8)$ & $33.3 \pm 18.0 \%$ & & $33.3 \pm 1.80 \%$ \\
\hline EP GII $(n=3)$ & $100.0 \%$ & & $100.0 \%$ \\
\hline PA GI $(n=9)$ & $100.0 \%$ & & $100.0 \%$ \\
\hline AS GII $(n=1)$ & $100.0 \%$ & & $100.0 \%$ \\
\hline
\end{tabular}

AS GII: Astrocytoma grade II, CMB: Classic Medulloblastoma, DNMB: Desmoplastic nodular medulloblastoma, EP GII: Ependymoma grade II, EP GIII: Ependymoma grade III, GTR: Gross Total Resection, LC/A MB: Large Cell Anaplastic Medulloblastoma, MO: No CSF seeding, M+: Positive CSF seeding, OS: Overall Survival, PFS: Progression-Free Survival, STR: Subtotal Resection, V4: Fourth Ventricle, *= Statistically significant. 
gradually over 1 month. The other five patients required tracheostomy, which was discontinued 2-4 months later. The univariate analysis showed a significantly higher risk of bulbar paralysis in patients who had undergone STR than in those undergone GTR $(p=0.034)$. Tumor size and histopathological type had no significant impact on developing bulbar palsy $(p=0.737$ and $p=0.116$ respectively).

\section{Outcomes}

\section{Risk stratification of medulloblastoma}

Medulloblastoma patients were divided into two groups: High-risk (HR) defined as M+ Chang stage (metastatic), age $<3$ years old and/or residual tumor $>1.5 \mathrm{~cm}^{2}$; and average/ standard-risk (SR): no metastasis or residual tumor. The SR patients had more favorable PFS than the HR patients (2-year PFS $85 \%$ vs. $21.5 \%, p=0.005)$. Nine of $13 \mathrm{HR}$ patients $(69.2 \%)$ experienced a relapse during the follow-up period, whereas two of 14 SR patients (14.3\%) relapsed (Table I).

\section{Disease progression and mortality}

Tumor metastasis (tumor seeding): the patients were classified into M0 (without CSF metastasis) or M+ (with CSF metastasis) Chang stages according to their CSF cytology and/or MRI of the cranio-spinal axis. The MO group had statistically significantly better PFS and OS than the M+ group (2-year PFS $93.3 \%$ vs. $18.7 \%, p<0.001)$. In total, 29 of $44(65.9 \%)$ patients had no metastasis (M0). Fifteen patients (34.1\%) had metastases, two of these patients had spinal seeding (enhanced nodules) before excision, 10 had positive CSF cytology, and three developed leptomeningeal dissemination later on. Four of the $14 \mathrm{CMB}$ patients $(28.6 \%)$, two of the five DNMB patients (40.0\%), all LC/A MB patients, and one of the three patients (33.3\%) with EP GIII had metastases. Time to metastasis was $0-15$ (mean: $0.80 \pm 1.821$ ) months.

Tumor recurrence: Three patients $(6.8 \%)$ had a tumor recurrence, two with EP GIII (recurrence 2 and 6 months after excision, respectively, the patient with early recurrence died 1 month later), and one patient with CMB (recurrence after 5 months). The time to recurrence was $2-6$ (mean: $4.33 \pm 2.082$ ) months.

Disease progression: Patients with pilocytic astrocytoma, AS GII, and EP GIl had no progression during the follow-up period and overall the best outcome. Thirteen patients $(30.2 \%)$ had disease progression: two patients had tumor recurrence, ten had tumor metastasis, and one had both.. These thirteen patients exhibited the following tumor types: four of fourteen CMB patients (28.6\%), two of five DNMB patients $(40.0 \%)$, five of eight LC/A MB patients (62.5\%), and two of three patients $(66.67 \%)$ with EP GIII. The time to progression in these patients was 2-20 months from excision (mean: 10.48 \pm 4.820 months).

Ten patients (23.3\%) died after variable periods of follow up, all had disease progression (one had tumor recurrence and metastasis, and nine had metastasis). Five of the eight patients with LC/A MB (62.5\%), one of the three patients with EP GIII (33.3\%), three of the 14 patients with CMB (21.4\%), and one of the five patients with DNMB $(20 \%)$ died. No patients with
PA GI, AS GII, and EP GIl subtype died during the follow up. Time to death was 2-20 (mean: $11.15 \pm 4.630$ ) months for all MB patients and 3- 18 (mean: $11.33 \pm 5.203$ ) months for ependymoma patients.

\section{Univariate survival analysis}

The estimated 2-year PFS and OS, using Kaplan-Meier statistics, for all histopathological types are listed in Table III.

Patients with CMB exhibited better PFS and OS than patients with DNMB (2-year PFS $66.0 \%$ vs. $60.0 \%$ ), and both CMB and DNMB patients had better PFS than LC/A MB and EP GIII patients, who had the poorest prognosis (2-year PFS 33.3\%, $\mathrm{p}=0.038$ ).

The estimated 2-year PFS and OS for all patients were $68.1 \%$ $\pm 7.4 \%$ (15.632 months) and $74.3 \% \pm 7.1 \%$ (16.755 months), respectively (Figure 1A, 2A). CSF seeding / metastasis $(p<0.001)$, HR group of MB $(p=0.005)$, subtotal resection of tumor (STR) $(p=0.020)$, and LC/A MB and anaplastic ependymoma $G$ III subtypes $(p=0.038)$ all had significant negative impact on PFS and OS, i.e., they are negative predictors (Figure 1B-E; 2B-E). Age, gender, and V4 floor infiltration had no impact on outcome (Figure 1F, 2F).

\section{Multivariate survival analysis}

Cox multivariate regression was used to determine the effect of important risk factors on OS and PFS. Subtotal resection $(p=0.036)$ was an independent negative predictor, whereas age, gender, brain stem involvement, metastasis, tumor size, and pathology had no predictive value.

\section{DISCUSSION}

The anatomy of the cerebellomedullary fissure was first depicted in 1922 by Matsushima et al. who suggested it as a potential route for approaching the fourth ventricle without neural tissue injury (16).

In our series of 44 patients, 37 patients (84.1\%) had GTR, seven patients $(15.9 \%)$ had STR. STR was largely due to brainstem involvement. Generally, we had no problem with tumor size or location in the superior part of the ventricle. GTR was $88.9 \%$ in Tomasello et al.'s case series $(25,26)$, and $82.0 \%$ in Han et al.'s series (10).

In our study, we found that larger tumors tend to extend and thin the inferior medullary velum and tela choroidea, in this way encouraging a less demanding and more extensive access to the tumor that provides early access to the floor of the ventricle. The same was also reported by Tomasello et al. $(25,26)$.

In our series, brainstem involvement was a major factor associated with STR $(p=0.008)$ in the 24 patients $(54.5 \%)$ whose tumors were invading or attached to the brainstem. GTR, meaning a complete resection or leaving a thin layer of tumor on the brain stem to avoid brainstem injury, could be achieved in $17(70.8 \%)$ of these patients, whereas the other seven patients $(29.2 \%)$ had STR. However, this problem may also occur when utilizing the transvermian route. Our results 
imply that tumor adherence to the brainstem is not an absolute contraindication for total tumor resection, which is consistent with Winkler et al.'s report (28).

Tomasello et al. also reported that large tumors and tumors extending to the upper third of the fourth ventricle are more surgically challenging and increase the risk of both STR and postoperative hydrocephalus (shunt dependency) $(25,26)$. These difficulties may also be encountered when choosing the transvermian route, but Tanriover et al. reported that the transvermian route provides a wider working angle to the rostral part of the fourth ventricle than the telovelar approach (24). Opposed to that, we did not find any problem with GTR of tumors extending to the rostral third of the fourth ventricle, because we routinely remove the posterior arch of the atlas, which provides a wider working angle. This finding is supported by Deshmukh et al.'s conclusion that the telovelar route together with removing of the posterior arch of the atlas results in a wider working angle to the upper third

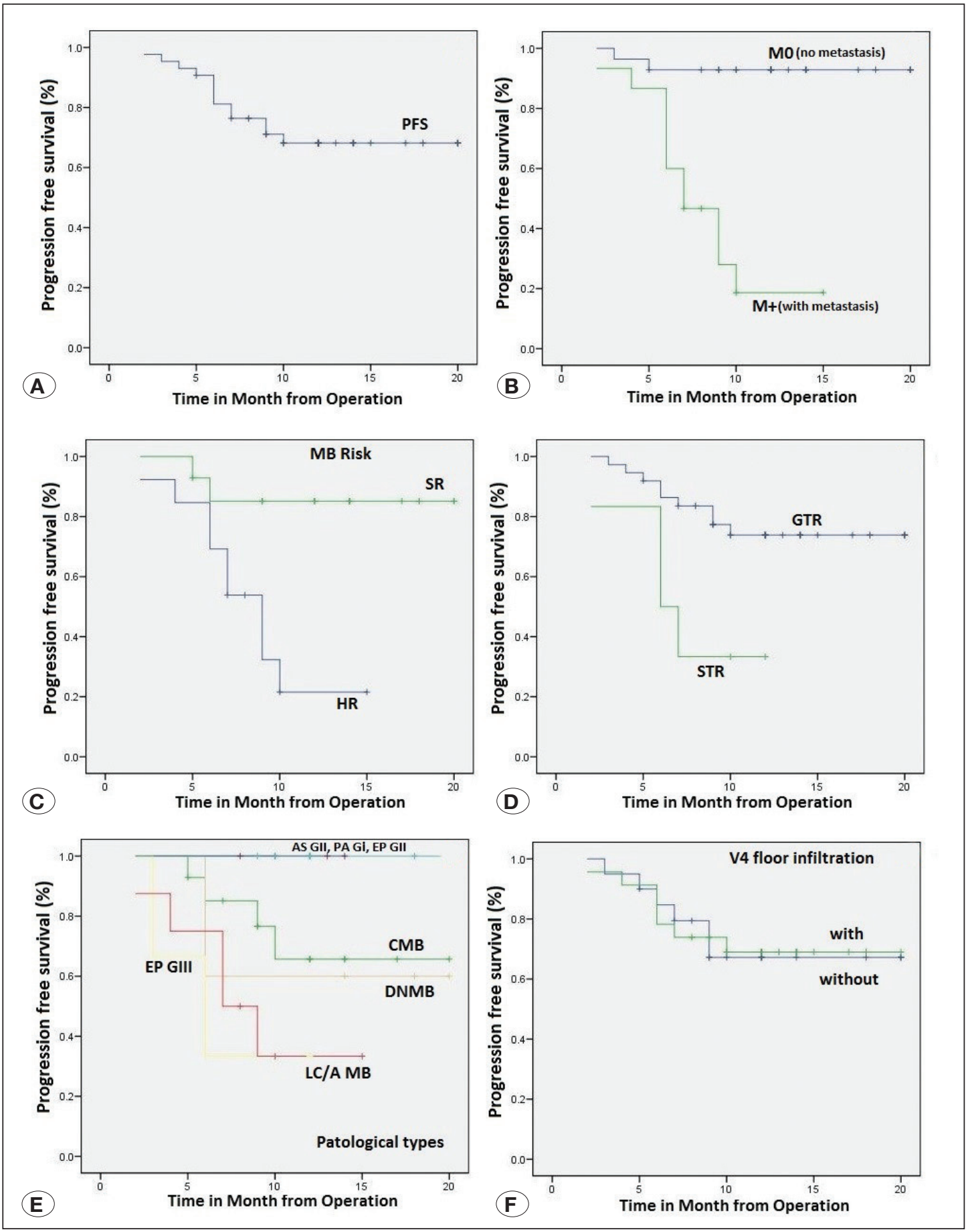

Figure 1: KaplanMeier estimation for PFS based on clinical factors: A) PFS in all cases; B) CSF seeding of tumor (MO versus $\mathrm{M}+)$; C) HR group of $M B$ versus SR group;

D) GTR versus STR; E) Pathological subtypes; F) Tumor location with V4 floor infiltration versus without infiltration. 
of the ventricle than the angle offered by the transvermian approach (4). Excision of parts of the cerebellar tonsils has been proposed as a solution to the limitations of the telovelar approach whenever the tumor is expansive or extends cranially and laterally (14). However, we believe that resection of the tonsils is not important in these cases.

We emphasize that the telovelar approach gives the advantage of early visualization and dissection of the interface between the tumor and the fourth ventricle floor, unlike the transvermian approach to the tumor from the back.

In our study, the incidence of CM was $13.6 \%$. It had been reported that the rate of $\mathrm{CM}$ can be as high as $30.0 \%$ with the telovelar approach $(10,29)$.

Gok et al., Han et al., and Matsushima et al. reported that the cases with $\mathrm{CM}$ all had undergone bilateral telovelar opening, whereas the occurrence of $\mathrm{CM}$ was improbable with unilateral

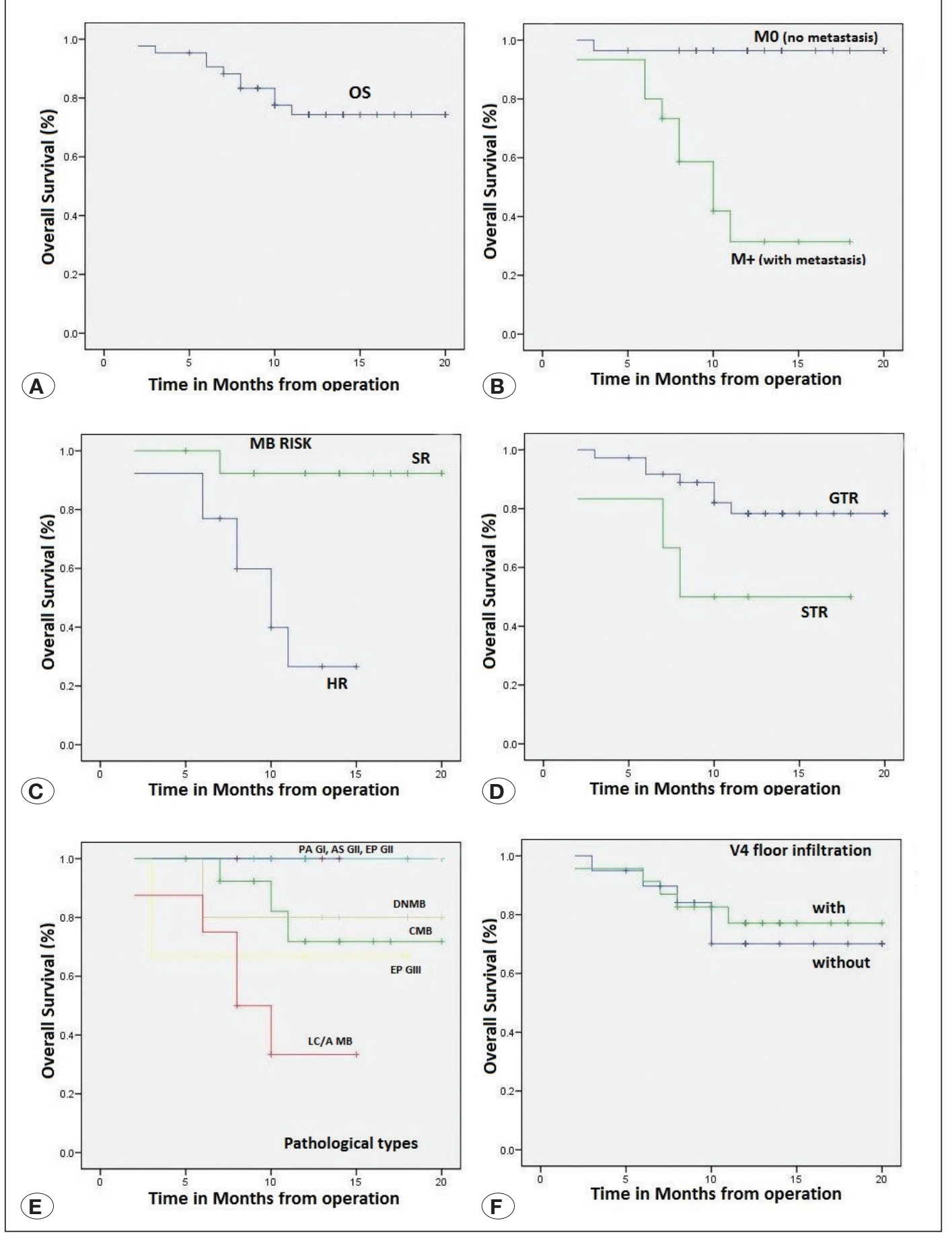

Figure 2: Kaplan-Meier estimation for OS based on clinical factors:

A) OS in all cases; B) CSF seeding of tumor (MO versus $\mathrm{M+})$; C) HR group of MB versus SR group; D) GTR versus STR; E) Pathological subtypes; F) Tumor location with V4 floor infiltration versus without infiltration. 
telovelar opening $(9,10,17)$. Contrary to that, Tomasello et al. used bilateral fissure opening and encountered no case of CM $(25,26)$. They proposed that the lack of CM in their study could be explained by the relatively higher mean age of their patients. They also suggested that cerebellar dysfunction may be avoided by wider fissure opening and limited cerebellar retraction. In our study, we used bilateral fissure opening and gentle retraction in almost all cases, but we had six cases (13.6\%) of CM. We can therefore only speculate on the cause of $\mathrm{CM}$ in these patients. It may be related to the low mean age of our patients ( 6.05 years) and/or the transient post-operative edema of the cerebellum and dentate nuclei as a result of surgical manipulation or cerebellar retraction. This was also reported by Wells et al., who noticed a significant edema of the cerebellum and the middle and superior cerebellar peduncles in the postoperative imaging of patients with CM (27). In our study, CM occurred 48-96 hours post-surgery, and recovery was associated with residual dysarthria, which was previously reported and referred to as syndrome of $\mathrm{CM}$ with subsequent dysarthria (5). In our study, all six patients who had CM had a pathology of $\mathrm{MB}$, and we therefore confirm that $\mathrm{MB}$ is a risk factor for $\mathrm{CM}$. This is consistent with other findings reported in the literature $(15,18,20,22)$.

In our study, we noticed a significantly higher risk of bulbar paralysis in patients who had undergone STR than in those undergone GTR $(p=0.034)$, which may be due to brainstem invasion rather than to STR itself.

In our series, the estimated 2-year PFS and OS of all patients were $68.1 \% \pm 7.4 \%$ (15.632 months) and $74.3 \% \pm 7.1 \%$ (16.755 months), respectively. This is relatively better than Jiang et al. who reported their 5 -year PFS and OS as $47.1 \% \pm$ $4.6 \%$ and $54.6 \% \pm 4.6 \%$, respectively (13).

We found that PFS and OS were better in patients who had GTR $(p=0.020)$, patients without CSF metastasis $(p<0.001)$, and in the SR group of MB patients $(p=0.005)$. This was also reported in other series $(3,7,13,23)$.

In our series, patients with pilocytic astrocytoma GI, astrocytoma GIl, and ependymoma Gll had no progression during the follow-up period and the overall best outcome. Patients with CMB exhibited a better PFS and OS than DNMB, and both $\mathrm{CMB}$ and $\mathrm{DNMB}$ had a statistically significant better PFS than LC/A MB and EP GIII patients, who had the worst prognosis $(p=0.038)$. This is consistent with the results of other clinical series $(6,8)$, which stated that MB is associated with the worst prognosis among posterior fossa tumors. Jiang et al. in their series of CMB, DNMB, and LC/A MB found a 5 -year PFS of $51.4 \%, 50.0 \%$, and $9.1 \%$, respectively (13).

The early outcome of patients is directly related to the surgical procedure and postoperative sequelae, whereas long-term outcome depends on several factors including diseaserelated morbidity and delayed effects of adjuvant therapies as described in other reports (12).

Recently, MB has been classified into four molecular types: $\mathrm{SHH}, \mathrm{WNT}$, type $\mathrm{C} / 3$, and type D/4 (19). Also, posterior fossa ependymoma has been classified into three molecular types: sub-ependymoma (PF-SE), PF-EPN-A, and PFEPN-B (1). Despite being similar histologically, all these molecular subgroups show a distinct behavior and prognosis, and we highly recommend performing a prospective study distinguishing between these molecular subgroups to draw definitive conclusions about their implications on outcomes.

\section{CONCLUSION}

The telovelar approach provides a number of merits and results in lower complication rates than the transvermian approach. It should therefore be the standard approach for fourth ventricle lesions. Early exposure of the interface between the tumor and the fourth ventricle floor, when possible, favors safer tumor resection. Whenever there is attachment of the tumor to the ventricle floor, safe resection leaving a thin layer of tumor on the brainstem is recommended to avoid brainstem injury. Wide opening of the cerebellomedullary space guarantees excellent exposure of the fourth ventricle cavity including lateral and superolateral recesses and obviates the need for excessive tonsillar retraction. This technique results in a lower risk of $\mathrm{CM}$ and other cerebellar dysfunctions. We recommend routine removal of the posterior arch of the atlas to gain a wider working angle, particularly to the deep rostral part of the ventricle. Tumor metastasis (CSF seeding), STR, HR group medulloblastoma, LC/A MB, and anaplastic ependymoma GIII are statistically significant negative prognostic factors.

\section{ACKNOWLEDGEMENT}

"The authors would like to thank Enago (www.enago.com) for the English language review."

\section{REFERENCES}

1. Archer TC, Pomeroy SL: Defining the molecular landscape of ependymomas. Cancer Cell 20:133-134, 2015

2. Calenbergh VF, De Laar VA, Plets C, Goffin J, Casaer P: Transient cerebellar mutism after posterior fossa surgery in children. Neurosurgery 37:894-898, 1995

3. Call JA, Naik M, Rodriguez FJ, Giannini C, Wu W, Buckner JC, Parney IF, Laack NN: Long-term outcomes and role of chemotherapy in adults with newly diagnosed medulloblastoma. Am J Clin Oncol 37:1-7, 2014

4. Deshmukh VR, Figueiredo EB, Crawford NR, Preul MC, Spetzler RF: Quantification and comparison of telovelar and transvermian approaches to the fourth ventricle. Neurosurgery 58 ONS Suppl 2:ONS 202-207, 2006

5. De Smet HJ, Baillieux H, Catsman-Berrevoets C, De Deyn PP, Mariën P, Paquier PF: Postoperative motor speech production in children with the syndrome of 'cerebellar' mutism and subsequent dysarthria: A critical review of the literature. Eur $\mathrm{J}$ Paediatr Neurol 11(4):193-207, 2007

6. Eberhart CG, Kratz JE, Schuster A, Goldthwaite P, Cohen KJ, Perlman EJ, Burger PC: Comparative genomic hybridization detects an increased number of chromosomal alterations in large cell/anaplastic medulloblastomas. Brain Pathol 12(1): 36-44, 2002 
7. Friedrich $\mathrm{C}$, von Bueren $\mathrm{AO}$, von Hoff $\mathrm{K}$, Kwiecien $\mathrm{R}$, Pietsch T, Warmuth-Metz M, Hau; P, Deinlein F, Kuehl J, Kortmann RD, Rutkowski S: Treatment of adult nonmetastatic medulloblastoma patients according to the paediatric HIT 2000 protocol: A prospective observational multicentre study. Eur J Cancer 49:893-903, 2013

8. Giangaspero F, Wellek S, Masuoka J, Gessi M, Kleihues P, Ongaki $\mathrm{H}$ : Stratifi cation of medulloblastoma on the basis of histopathological grading. Acta Neuropathologica 112:5-12, 2006

9. Gok A, Alptekin M, Erkutlu I: Surgical approach to the fourth ventricle cavity through the cerebellomedullary fissure. Neurosurg Rev 27:50-54, 2004

10. Han S, Wang Z, Wang Y, Wu A: Transcerebellomedullary fissure approach to lesions of the fourth ventricle: Less is more? Acta Neurochir 155:1011-1016, 2013

11. Holmes G: The Croonian lectures on the clinical symptoms of cerebellar disease and their interpretation. Lecture II. 1922. Cerebellum 6(2):148-153; discussion 141,2007

12. Hoppe-Hirsch E, Renier D, Lellouch-Tubiana A, Sainte-Rose C, Pierre-Kahn A, Hirsch JF: Medulloblastoma in childhood: Progressive intellectual deterioration. Child's Nerv Syst 6:6065, 1990

13. Jiang T, Zhang Y, Wang J, Du J, Ma Z, Li C, Liu, Zhang Y: Impact of tumor location and fourth ventricle infiltration in medulloblastoma. Acta Neurochir 158:1187-1195, 2016

14. Jittapiromsak P, Little AS, Deshmukh P, Nakaji P, Spetzler RF, Preul MC: Comparative analysis of the retrosigmoid and lateral supracerebellar infratentorial approaches along the lateral surface of the pontomesencephalic junction: A different perspective. Neurosurgery 62(5 Suppl 2):ONS279287, discussion ONS287-288, 2008

15. Kuplei S, Bilginer B, Buyukpamukcu M: Posterior fossa syndrome after posterior fossa surgery in children with brain tumors. Pediatric Blood Cancer 56(2):206-210, 2011

16. Matsushima T, Fukui M, Inoue T, Natori $Y$, Baba T, Fujii K: Microsurgical and magnetic resonance imaging anatomy of the cerebello-medullary fissure and its application during fourth ventricle surgery. Neurosurgery 30:325-330, 1992

17. Matsushima $T$, Inoue $T$, Inamura $T$, Natori $\mathrm{Y}$, Fukui $\mathrm{M}$ : Transcerebellomedullary fissure approach with special reference to methods of dissecting the fissure. J Neursurg 94:257-264, 2001

18. Mei C, Morgan AT: Incidence of mutism, dysarthria and dysphagia associated with childhood posterior fossa tumour. Childs Nerv Syst 27:1129-1136, 2011
19. Northcott PA, Korshunov A, Witt H, Hielscher T, Eberhart CG, Mack S, Bouffet E, Clifford SC, Hawkins CE, French P, Rutka JT, Pfister S, Taylor MD: Medulloblastoma comprises four distinct molecular variants. J Clin Oncology 29:1408-1414, 2011

20. Pollack IF: Neurobehavioral abnormalities after posterior fossa surgery in children. Int Rev Psychiatry 13(4):302-312, 2001

21. Pollack IF, Polinko P, Albright AL, Towbin R, Fitz C: Mutism and pseudobulbar symptoms after resection of posterior fossa tumors in children: Incidence and pathophysiology. Neurosurgery 37:885-893, 1995

22. Reed-Berendt R, Phillips B, Picton S, Chumas P, Warren D, Livingston JH, Hughes E, Morrall MC: Cause and outcome of cerebellar mutism: Evidence from a systematic review. Childs Nerv Syst 30:375-385, 2014

23. Sala F, Talacchi A, Mazza C, Prisco R, Ghimenton C, Bricolo A: Prognostic factors in childhood intracranial ependymomas: The role of age and tumor location. Pediatr Neurosurg 28:135142,1998

24. Tanriover N, Ulm AJ, Rhoton AL Jr, Yasuda A: Comparison of the transvermian and telovelar approaches to the fourth ventricle. J Neurosurg 101:484-498, 2004

25. Tomasello F, Conti A, Angileri FF, Cardali S: Telovelar approach to fourth-ventricle tumours: How I do it. Acta Neurochir (Wien) 157(4):607-610, 2015

26. Tomasello F, Conti A, Cardali S, La Torre D, Angileri Filippo F: Telovelar approach to fourth ventricle tumors: highlights and limitations. World Neurosurg 83(6):1141-1147, 2015

27. Wells EM, Khademian ZP, Walsh KS, Vezina G, Sposto R, Keating RF, Packer RJ: Post-operative cerebellar mutism syndrome following treatment of medulloblastoma: Neuroradiographic features and origin. J Neurosurg Pediatrics 5:329 334,2010

28. Winkler EA, Birk $H$, Safaee $M$, Yue JK, Burke JF, Viner JA, Pekmezci M, Perry A, Aghi MK, Berger MS, McDermott MW: Surgical resection of fourth ventricular ependymomas: Case series and technical nuances. J Neurooncol 130:341-349, 2016

29. Zaheer SN, Wood M: Experiences with the telovelar approach to fourth ventricular tumors in children. Pediatr Neurosurg 46:340-343, 2010

30. Ziyal IM, Sekhar LN, Salas E: Subtonsillar transcerebellomedullary approach to lesions involving the fourth ventricle, the cerebellomedullary fissure and the lateral brainstem. Br J Neurosurg 13:276-284, 1999 